\title{
MUJER Y POLÍTICA EN EL MUNDO ÁRABE. UN ESTADO DE LA CUESTIÓN
}

\author{
YOLANDA AIXELÁ Y ANA I. PLANET \\ Universidad de Alicante
}

\section{INTRODUCCIÓN}

El mundo árabe está constituido por un amplio conjunto de países que van desde Marruecos en el occidente hasta Irak en el oriente. Pese a la aparente sencillez de la definición geográfica, la complejidad asociada a la presencia del islam como religión de la mayoría de sus habitantes hace confundir bajo esa etiqueta a estados vecinos no árabes pero de mayoría musulmana -Irán, Turquía y algunas repúblicas ex-soviéticas- y dificulta en ocasiones el estudio de la región, aunque también enriquezca el debate sobre las diferentes cuestiones por la posibilidad comparativa que aporta. A esta dificultad se añade la existencia de numerosos términos para designar la región y una falta de definición de sus límites en algunos trabajos ${ }^{1}$.

1. El término Middle East (traducido al castellano como Oriente Medio) fue acuñado por el historiador americano A. T. Mahan en 1900 como parte de su análisis de la región y utilizado por los británicos durante la I Guerra Mundial. Véase SAVory, R. M. (ed.): Introduction to Islamic Civilisation, Cambridge, Cambridge University Press, 1984. Se trata en esta concepción de un término geoestratégico en el que quedarían incluidos países del Norte de África (Libia, Egipto y Sudán y también para algunos Etiopía, Somalia e incluso la totalidad del cuerno de África), países de Asia (Arabia Saudí y el resto de países de la Península Arábiga, así como Israel, Jordania, Siria, Líbano, Irak, Irán, Afganistán y Pakistán) y Turquía. En algunos casos podremos encontrar incluidos en esta región Chipre, algunas zonas de los Balcanes y las cinco repúblicas ex-soviéticas meridionales. Es interesante señalar que en la bibliografía anglosajona, al término Middle East se añade North Africa para incluir en la misma región Túnez, Argelia, Marruecos y Mauritania, dando lugar al área de estudio MENA (Middle East and North Africa). Para el antropólogo Dale Eickelman, autor de The Middle East and Central Asia. An Anthropological Approach (New Jersey, Prentice-Hall, 1989), traducido al castellano como Antropología del mundo musulmán (Barcelona, Bellaterra, 2003), este término se emplearía para definir la zona que va desde Rabat hasta Teherán y pese a su marcado carácter eurocéntrico se habría impuesto y normalizado su uso, siendo utilizado incluso por los especialistas de los propios países concernidos. El término Moyen Orient, inicialmente traducción del francés del anterior, se confunde en ocasiones en su utilización con el más amplio de Proche Oriente (Oriente Próximo) -que englobaría Chipre, Israel y Turquía además de Egipto, la Península Arábiga y los países asiáticos considerados por la terminología en inglés-y precisa, como en 
Por otra parte, la heterogeneidad social y la pluralidad religiosa que encontramos en las sociedades árabes contemporáneas ha dado lugar a ciertos enfoques basados en la consideración de las sociedades árabes como sociedades desmembradas, constituidas por grupos o sectas religiosas enfrentadas entre sí o por minorías con dificultades de relación. Plantear la existencia de elementos comunes como la lengua, la cultura, la geografía o la historia y otros que marcan diferencias -como son las formas estatales, las solidaridades grupales, las divisiones sectarias o localismos- nos ayuda a analizar unas sociedades dinámicas cuyas bases identitarias podrían encontrarse en el binomio islam-arabidad planteado en perspectiva crítica, como aporta el sociólogo tunecino Hichem Djait ${ }^{2}$. Para entender lo que el islam significa en la región, como señaló en 1925 el pensador Ali Abd al-Ráziq, habría que recurrir a la historia y al estudio del momento en el que surge el islam y se inicia su expansión para darnos cuenta de hasta qué punto éste ha constituido un elemento de unificación de los pueblos árabes. Sin embargo, no debe considerarse que esa unidad a través del islam se realiza en términos políticos sino en términos religiosos-"una unidad en la fe y la doctrina religiosa, no una unidad estatal según las representaciones del poder temporal $\aleph^{3}$-. Los árabes después de la llegada del islam han continuado formando pueblos y entidades políticas distintas, aunque el islam les haya dado instituciones y costumbres semejantes. El desarrollo de los Estados nación a lo largo del siglo XX terminará de forjar esas diferencias.

Es quizá la situación de la mujer uno de los puntos en los que se ha tendido a encontrar mayor similitud en las sociedades árabes contemporáneas. Una imagen común a estas sociedades es que mantienen a las mujeres en un estado de sumisión y de falta de independencia respecto a los hombres. Sin embargo, las mujeres en el mundo árabe han venido participando de distintas maneras, con mayor o menor visibilidad, en la esfera política, hecho que no siempre se ha percibido y recogido por los científicos sociales. Probablemente la causa es que los análisis adolecían de algunos prejuicios: por un lado, incorporaban una mirada sesgada por algunos presupuestos considerados como indiscutibles (el patriarcado, la complementariedad sexual propugnada por el Corán, el controvertido uso del hiyab...); por el otro, se producía una constante comparación entre el caso árabe y el europeo sin contar que sus tradiciones históricas eran distintas. En cualquier caso, en este texto presentaremos una aproximación a algunos de los trabajos desarrollados sobre el particular con el interés de clarificar en lo posible algunos de los debates existentes sobre «mujeres y política» en el contexto de los estados árabes contemporáneos y de su actual evolución.

el caso anterior, de la inclusión de los países del Magreb para completar el conjunto del mundo árabe. Véase Lacoste, Yves (dir.): Dictionnaire de géopolitique, París, Flammarion, 1993.

2. Véase DJAïT, Hichem: La personalidad y el devenir araboislámicos, Madrid, Libertarias, 1996.

3. Véase AbDDERRAzIK, Ali: L'islam et les fondements du pouvoir, Casablanca, Le Fennec, 1994 (traducción y edición a cargo de Abdou FILALY-ANSARY sobre la edición original de 1925). 


\section{LAS POSIBILIDADES DE PARTICIPACIÓN: DEMOCRATIZACIÓN- LIBERALIZACIÓN EN EL MUNDO ÁRABE Y LA ECLOSIÓN DE LA SOCIEDAD CIVIL}

Un debate central para aquellos que analizan la situación política de los estados árabes y las posibilidades de éxito de los cambios que en los sistemas políticos árabes están teniendo lugar desde la década de los noventa tiene que ver con la dificultad de considerarlos como democráticos. Insertos en marcos estatales cuyas instituciones adolecen de falta de representatividad y capacidad de decisión y en los que su representatividad es puesta en duda, los cambios en la relación entre el Estado y el ciudadano que se vienen dando desde estas instituciones son más respuesta a las diferentes crisis que éste sufre, buscan fundamentalmente su permanencia y rara vez proponen reformas profundas o una transición hacia otro sistema. Pese a lo limitado de su alcance, estos procesos existen y tienen sus efectos en las sociedades de la región. Para analizarlos y dar cabida a las consecuentes modificaciones en las estructuras cabría, quizás, recurrir a la distinción entre "procesos de liberalización política» y "procesos de democratización». Así, si los primeros se caracterizan por la ampliación por parte del Estado de los espacios públicos, por el aumento de la participación de la sociedad civil mediante la expresión de sus necesidades y por el planteamiento de demandas a las instituciones -todo ello acompañado de un mayor reconocimiento y protección de las libertades públicas y civiles- los procesos de democratización, por su parte, deberían ir necesariamente acompañados de una ampliación de la participación como instrumento para que los ciudadanos puedan ejercer de forma colectiva un cierto grado de control sobre las políticas públicas, no sólo en el planteamiento de demandas sino también en el desarrollo de los instrumentos para su realización y posterior control. Este aumento de la participación del ciudadano se desarrolla fundamentalmente a través de la elección en las urnas de representantes en las diferentes instancias de ejercicio del poder ${ }^{4}$.

En el debate teórico sobre liberalización y democratización en el mundo árabe que está presente en la mayoría de escritos de los especialistas dedicados a la región desde ella y desde fuera de ella se encuentra otro debate que también afecta a la cuestión de la mujer, como es el hecho de que nos encontramos ante una región definida por ser el islam la religión mayoritaria de sus poblaciones así como por el carácter islámico de algunos de sus regímenes y la consecuente cuestión de la compatibilidad o incompatibilidad entre el islam y las posibilidades de desarrollo de una democracia. Aquellos que consideran la existencia de una incompatibilidad en este desarrollo encuentran uno de los condicionantes que lastran el mantenimiento de un sistema democrático en la teoría del poder en el islam en época clásica y el consecuente modelo de ciudadano-creyente

4. De las diferentes experiencias democratizadoras y sus límites hay interesantes reflexiones en Korany, Bahgat; Brynen, Rex y Noble, Paul (eds.): Political liberalization and democratization in the Arab World: Comparative Experiences, Londres, Boulder, 1998. 
que sustenta ${ }^{5}$. También se apunta en algunos trabajos hacia lo que algunos autores califican de "excepcionalidad democrática» en el mundo árabe e islámico que parte de la caracterización del cuerpo social en los países de la región como primordialista -basado en lazos tribales, clánicos o sectarios-, en el que se desarrollan modos de autoritarismo y sumisión al poder que coexisten con principios de participación a través de la Shura o principio de consulta, o con el lazo de compromiso existente entre gobernado y gobernante a través de la baia, lo que daría lugar a unas formas específicas de inserción del ciudadano en el sistema que no serían democráticas stricto $s e n s 0^{6}$. Dentro de estos debates resulta también de interés el que se refiere a la pertinencia de emplear el término de "cultura política arabo-islámica» como una variable única en la que se englobarían los aspectos antes mencionados, con la prevención de que un término así implica -en nuestra opinión su utilización puede servir de cajón de sastre que dificulte al análisis de los distintos elementos de tal variable- ${ }^{7}$.

Tras estos debates, e iluminando especialmente lo que se refiere a la mujer y su participación en la esfera de lo político, se encuentra una reflexión esencial sobre la relación entre las condiciones socioeconómicas y los procesos de democratización, así como sobre el desarrollo de la sociedad civil como requisito para el éxito de estos procesos que se ven afectados por la situación internacional desde los atentados contra las Torres Gemelas y la nueva política de lucha contra el terrorismo internacional que ha paralizado en buena medida su desarrollo.

\section{UNA SOCIEDAD CIVIL PARA CADA ESTADO: LOS LÍMITES DE UN CONCEPTO}

La dificultad para desarrollar un análisis de la sociedad civil en los países de la región supera la cuestión de dilucidar si se trata o no de un marco teórico importado. La utilización del concepto de sociedad civil en tanto que concepto en continua redefinición así como la determinación de los elementos, grupos o tendencias que la constituyen son aspectos sobre los que no existe consenso en el mundo árabe ${ }^{8}$. En la base de cualquier reflexión al respecto se encuentra, además,

5. Véase al respecto el ensayo de CHARFI, Mohamed: Islam y libertad. El malentendido histórico, Granada, Almed, 2001; Gardet, Louis: La cité musulmane. Vie sociale et politique, París, Librairie Philosophique J. Vrin, 1954 y GHalioun, Burhan: Islam y política. Las traiciones de la modernidad, Barcelona, Bellaterra, 1999 (edición original en francés de 1997).

6. Véase Anderson, Lisa: «Democracy in the Arab World: A Critique of the Political Culture Approach» en Rex Brynen, Bahgat Korany y Paul Noble (eds.): Political liberalization and democratization in the Arab World: theoretical perspectives, Londres, Boulder, 1995, pp. 73-92.

7. Véase Kedourie, Elie: Democracy and arab political culture, Londres, Frank Cass, 1994 y Hudson, Michael: Arab Politics. The Search for Legitimacy, Londres, Yale University Press, 1977.

8. Esto tiene reflejo, incluso, en la terminología empleada en lengua árabe. Al término más comúnmente utilizado de al-muchtama al-madani se une la posibilidad de emplear al-muchtama al-ahli. El politólogo Nazih Ayubi apuesta por el segundo de los términos por considerar que si bien almadani está más próximo al concepto «civil» parece referirse tan sólo a organizaciones seculares, mientras que al-ahli incorpora organizaciones de inspiración religiosa islámica o de otro orden que, según el autor, pueden incluirse dentro de la sociedad civil. Véase Ayubi, Nazih: Política y sociedad en Oriente próximo. La hipertrofia del estado árabe, Barcelona, Bellaterra, 1998 (edición original en inglés de 1995). 
el modelo de Estado tomado en consideración y las posibilidades de desarrollo de tales grupos. La referencia es clara cuando se exponen las limitaciones en el desarrollo de una sociedad civil en un régimen autoritario.

La cuestión sobre la existencia o no de una sociedad civil en el mundo árabe es un debate abierto, tanto en su dimensión teórica-investigadora como en su dimensión práctica-política. Sin ánimo simplificador se podrían mencionar tres posturas principales al respecto, desde aquellos que no consideran útil el concepto para analizar los grupos y tendencias desarrolladas en la sociedad árabe contemporánea, partiendo para ello de la propia caracterización del Estado en la región, a los que sugieren otros enfoques más adecuados que tengan en cuenta el desarrollo histórico y cultural o algunos que proponen aceptar el concepto, pero añadiéndole organizaciones y grupos que no estaban siendo considerados como tales en trabajos sobre las sociedades europeas o americanas.

Como decíamos, la posibilidad de desarrollo de una sociedad civil guarda estrecha relación con el modelo de Estado y con las lógicas de inclusión de grupos sociales desarrolladas por él. En el estudio del Estado y de la sociedad civil algunos autores -entre los que destacaremos a Nazih Ayubi y Gasán Salamé-, señalan que entre los requisitos que favorecen la creación de grupos susceptibles de ser considerados como sociedad civil está la existencia de una sociedad con divisiones de clase en la que los individuos tengan conciencia de clase, con capacidad de los ciudadanos para expresar intereses y buscar su consecución al margen de las pertenencias primarias -familia, redes clientelares, lealtades tribales...- así como una relación del Estado con los ciudadanos y con los grupos en la que se incluya la posibilidad de que éstos disientan tanto de las políticas estatales como de las opiniones mayoritarias?.

La relación del Estado con estas organizaciones, así como su participación en su surgimiento y mantenimiento no es igual en todos los $\operatorname{casos}^{10}$. En la región encontramos países en los que se facilita la libertad de asociación en diferentes ámbitos y que están inmersos en los llamados procesos de liberalización política, como puedan ser Túnez, Jordania o Egipto y donde las asociaciones sirven de semillero y de campo de entrenamiento de expresión pública desde hace varias décadas -con las dificultades de expresión que se vienen dando en los últimos años-. En otros muchos países la libertad de asociación viene limitada por el partido único o cualquier otro modo de organización global, como es el caso de Siria -a través del partido Baaz- o Libia, y en otros tantos -a la cabeza de los cuales se encontraría Arabia Saudí- no existe libertad de asociación, ni en la forma de partidos políticos ni de asociaciones de ningún tipo. Algunos autores han querido ver en formas tradicionales de asociacionismo en estos países -como los diwániyya kuwaitíes- prácticas y actitudes propias de la sociedad civil ${ }^{11}$.

9. Véase Salamé, Ghassan (dir.): Démocratie sans démocrates: Politiques d'ouverture dans le monde arabe et islamique, París, Fayard, 1994.

10. Véase Moussalli, Ahmed: «Modern islamic fundamentalism discourse» en Augustus Richard Norton: Civil society in the Middle East, Leiden, Brill, 1995, volumen 1, pp. 79-119.

11. Hicks, Neil y NAJJAR, Ghanim (1995): «The utility of tradition: civil society in Kuwait» en Augustus Richard Norton: Op. cit., volumen 1, pp. 186-213. 


\section{MUJER Y POLÍTICA: UN ESTADO DE LA CUESTIÓN}

Como afirmábamos, la relación entre "mujeres» y "política» en el mundo árabe ha venido sufriendo algunas distorsiones fruto de algunas premisas erróneas manifestadas en buena parte de la producción europea y anglosajona, y por qué no, también arabo-musulmana. De hecho, se ha tendido a plantear una construcción de género en estas sociedades que partía de la existencia de una división complementaria de actividades según el sexo que conllevaba un estricto reparto espacial. Según éste, a las mujeres les atañían las responsabilidades domésticas y familiares del espacio privado, y a los hombres todas aquellas actividades que se desenvolvían en el espacio público -responsabilidades laborales, políticas, etc. ${ }^{12}$.

Este presupuesto, que no siempre se cumplía en la mayoría de las sociedades arabo-musulmanas del siglo XX, a pesar de ser paradigmático, colaboró por un lado en la invisibilidad de los poderes y estrategias femeninas en los diversos ámbitos sociales de los diferentes países, al tiempo que reforzaba una imagen masculina que caracterizaba a los hombres por su responsabilidad comunitaria: las mujeres no podían tener una perspectiva social amplia que fuese capaz de discernir los intereses de todo el grupo porque ésta era una característica eminentemente masculina; las mujeres no podían "pensar» más allá de las contingencias de su grupo familiar. Es por ello que parte de la producción científica desarrollada sobre "mujeres y política» normalmente obvió la participación de las mujeres en esta esfera social: se manifestaba una especie de ceguera permanente del científico social sobre las posibilidades que las mujeres arabomusulmanas tenían de interferir para transformar su realidad sociopolítica, en parte también porque se esperaba una influencia y representatividad cercana a la existente en los países europeos.

Fueron diversas las premisas sobre las que se construyó y se reafirmó esa imagen de pasividad e impotencia femenina. Por un lado, destaca que algunos defendían la existencia de un férreo patriarcado que negaba a las mujeres la posibilidad de participar en ninguna esfera social por hallarse sometidas y subordinadas a los hombres ${ }^{13}$. De hecho, esta propuesta venía avalada por la existencia generalizada de unos códigos jurídicos restrictivos para las mujeres -por ejemplo, la figura del wali como representante femenino en el matrimonio, permiso marital para obtener un trabajo en la economía formal- respecto a la extensión de los derechos masculinos -por ejemplo, la poliginia, salvo en

12. Véase Rodríguez Mellado, Inocencia: "Notas sobre la evolución social de la mujer egipcia», Cuadernos de Estudios Africanos, 17 (1952), pp. 37-48, para conocer el discurso colonial al respecto. Posicionamientos más recientes son los de El-KhaYat, Ghita: Le monde arabe au féminin, París, L'Harmattan, 1988 y Ruzz de Almodovar, Caridad: Historia del Movimiento Feminista Egipcio, Granada, Universidad de Granada, 1989.

13. Aunque los especialistas que mantendrían un posicionamiento similar serían numerosos, cabe destacar por su reconocida trayectoria a MerNISSI, Fátima: «Arab Women's Rights and the Muslim State in the Twenty-first Century: Reflections on Islam as Religion and State» en Faith \& Freedom. Women's human rights in the muslim world, London/New York, I. B. Tauris Publishers, 1995, pp. $33-50$. 
Túnez- ${ }^{14}$. Por otro lado, había una sistemática negación de las mujeres en el espacio sociopolítico evidenciado por su obligación de vestir un hiyab que las colectivizaba y reflejaba su carencia de derechos individuales ${ }^{15}$.

Además, cabe añadir que algunos de los trabajos consideraban, de una u otra manera, que la "modernidad política» se equipararía inevitablemente con un cierto "progreso", que debía construir a su vez, indefectiblemente, la emancipación femenina desde los parámetros de una parte del feminismo desarrollado en Europa: emancipación femenina = equidad sexual ${ }^{16}$, ello sin tener en cuenta que en algunos países árabes como Marruecos se está desarrollando un feminismo emancipatorio junto con un feminismo islamizante ${ }^{17}$.

En cualquier caso, aunque buena parte de la producción en las ciencias sociales se orientó, salvo escasas excepciones, hacia esa interpretación, las aportaciones de la década de los noventa destacaron la participación e influencia de las mujeres en el ámbito político ${ }^{18}$, una participación femenina que en el mundo árabe estaba, y aún está, plagada de claroscuros.

14. Véase Ruiz de Almodóvar, Caridad: «El código marroquí de estatuto personal y su reforma de 1993», en G. Martín Muñoz (ed.): Mujeres, democracia y desarrollo en el Magreb, Madrid, Pablo Iglesias, 1995, pp. 29-35 y, también, Feliu, Laura y Ramírez, Ángeles: «Mujeres y derechos humanos en el Magreb», Quaderns de la Mediterrània, 2-3 (2000), pp. 63-80. No obstante, este último trabajo citado conecta el Código Familiar con los movimientos feministas.

15. Véase Mernissi, Fátima: El miedo a la modernidad, Madrid, Ed. del Oriente Medio y el Mediterráneo, 1992.

16. Por ejemplo, Crinon, Monique: «Des femmes en mouvement pour la conquête de leurs droits», en C. Lacoste-Dujardin e Y. Lacoste (comp.): L'etat du Maghreb, Casablanca, Najah el Jadida, 1991, p. 221; BeLARBI, Aïcha: «Femme et sociéte civile: Reflexions sur le cas du Maroc», en M. Grazia y D. Barbalarga (eds.): Droits de citoyenneté des femmes au Maghreb, Casablanca, Le Fennec, 1997, pp. 249-272 y FerChiou, Sophie: «Feministe d'etat en Tunisie. Ideologie dominante et resistance féminine» en Femmes, culture et société au Maghreb. Femmes, pouvoir politique et dévelopement, Casablanca, Afrique-Orient, Vol II, 1996, pp. 119-140.

17. Véase Aixela Cabré, Yolanda: Mujeres en Marruecos. Un análisis desde el parentesco y el género, Barcelona, Bellaterra, 2000.

18. Véase Ferchiou, Sophie: "Organisation sociale et participation des femmes a la vie publique en Tunisie", Annuaire de l'Afrique du Nord, Centre Nationel du Recherche Scientifique, XXVI (1987), pp. 433-449; KandiYotI, Deniz: «Introduction», en D. Kandiyoti (comp.): Women, Islam \& State, London, Mcmillan, 1991, pp. 1-21; Dialmy, Abdessamad: Feminisme soufi, Casablanca, Afrique Orient, 1991; KandiYoti, Deniz: «Women, Islam and the State: A Comparative Approach», en R. I. Cole (ed.): Comparing Muslim Societies, Michigan, The University of Michigan Press, 1992, pp. 237-261; Ahmed, Leila: Women and Gender in Islam: Roots of Modern Debate, New Haven, Yale University Press, 1992; EL-KHAYAT, Ghita: Le Maghreb des femmes. Les femmes dans l'U.M.A, Casablanca, EDIF, 1992; DAoud, Zakya: Féminisme et politique au Maghreb, París, Eddif, 1993; DAOUD, Zakya: "Les associations féministes maghrébines et la conférence de Pekin», Annuaire de l'Afrique du Nord, XXXIV (1995), pp. 899-906; LAZREG, Marnia: The eloquence of silence: Algerian women in question, New York, Routledge, 1994; KandYoti, Deniz: "Contemporary Feminist Scholarship and Middle East Studies» en Deniz Kandiyoti (ed.): Gendering the Middle East, London, I.B.Tauris, 1996, pp.1-27; Perez Beltran, Carmelo: «Entre erradicación y diálogo: mujeres argelinas, Miscelánea de Estudios árabes y hebráicos, Sección Árabe-Islam, 45 (1996), pp. 203-232; BELARBI, Aïcha: «Femmes et société civile: Reflexions sur le cas du Maroc» en Droits et citoyennetté des femmes au Maghreb, Casablanca, Le Fennec, 1997, pp. 249-272; Al-Ali, Nadje: Secularism, Gender and the State in the Middle East: The Egyptian Women's Movement, Cambridge, Cambridge University Press, 2000; KandiYoti, Deniz: "Algunas cuestiones incómodas sobre las mujeres y 
Es por ello que algunos científicos sociales sugirieron la necesidad de debatir sobre la invisibilidad femenina en la esfera política en el mundo árabe, proporcionando un interesante contrapunto a ese tipo de explicaciones totalizadoras ${ }^{19}$. En el fondo del debate, la discusión de si las sociedades arabo-musulmanas, por sus características particulares androcéntricas y comunitarias, difícilmente podrían albergar la participación sociopolítica de las mujeres: islam $\neq$ democracia; islam $\neq$ sociedad civil.

\section{MUJERES AUSENTES: DISCURSOS SOBRE LA NO PARTICIPACIÓN DE LAS MUJERES EN LA ESFERA POLÍTICA}

Buena parte de los trabajos que inicialmente se desarrollaron sobre la participación política de las mujeres pusieron de relieve su ausencia e invisibilidad con distintos argumentos.

El primero de estos argumentos fue el patriarcado. Autores como ZenieZiegler o Kandiyoti ${ }^{20}$ definieron el mundo árabe como patriarcal. El patriarcado es un sistema político-social que fue extensamente trabajado por buena parte de los antropólogos evolucionistas de finales del XIX y principios del XX (por ejemplo, Johan Jakob Bachofen o Robertson-Smith para el mundo árabe). Según sus escritos, se trataba de sociedades en las que las mujeres estaban completamente supeditadas a los hombres. El problema de esta premisa es que era tan homogénea que impedía observar las aportaciones y poderes que las mujeres tenían en las diferentes esferas sociales. El desarrollo de la antropología permitió desmitificar este sistema político a través de diferentes teorías y trabajos etnográficos, lo que no obvió que algunos científicos sociales aplicaran este modelo a las sociedades arabo-musulmanas, incluso en trabajos más o menos recientes ${ }^{21}$. Por tanto, la defensa de la existencia de un patriarcado en el mundo árabe constituye una de las primeras causas de la invisibilidad política femenina: ¿cómo podría defenderse la influencia social de las mujeres arabo-musulmanas si aparentemente no tienen capacidad de acción?

El segundo argumento que avalaba tal planteamiento era que el Islam enunciaba una construcción de género basada en una complementariedad sexual que

la modernidad en Turquía» en L. Abu-Lughod, (ed): Feminismo y modernidad en Oriente Próximo, Madrid, Cátedra, 2002, pp. 395-420.

19. Véase Badran, Margot: Feminists, Islam and Nation. Gender and the Making of Modern Egypt, Princenton, Princenton University Press, 1995; ABu-Lughod, Lila: "Zones of Theory in the Anthropology of the Arab World», Ann. Rev. Anthropology, XVIII (1989), pp. 267-306 y ABU-LUGHod, Lila: «Introducción. Anhelos feministas y condiciones postcoloniales» en L. Abu-Lughod, (ed.): Feminismo y modernidad..., op. cit., pp. 13-56.

20. Zenie-Ziegler, Wedad: La face voilée des femmes d'Egypte, París, Mercure de France, 1985; Kandiyoti, Deniz, «Patterns of Patriarchy: Notes for an Analysis of Male Dominance in Turkish Society», en S. Tekeli (ed.): Women in Modern Turkish Society, Londres, Zed Books, 1995, pp. 306-318; Kandiyoti, Deniz: «Reflections on the Politics of Gender in Muslim Societies: from Nairobi to Beijing» en Faith \& Freedom. Women's human rights in the muslim world, London/New York, I. B. Tauris Publishers, 1995, pp. 19-32.

21. Como es el caso, por ejemplo, de Lacoste-Dujardin, Camille: Las madres contra las mujeres. Maternidad y patriarcado en el Magreb, Madrid, Cátedra, 1993. 
partía de que los hombres eran proveedores y protectores de la familia, y las mujeres madres y esposas. Está claro que si se revisa el Corán como fuente principal del islam hallaremos suras que legitimarían estas propuestas. No obstante, la antropología que fundamenta parte de sus resultados en el análisis local permite vislumbrar y demostrar la enorme distancia existente entre la teoría y la práctica social, hecho sobre lo que el propio Bourdieu ${ }^{22}$ teorizó certeramente: que se propugne una construcción de género determinada no implica que en cada país haya centenares de miles de familias que en su vida cotidiana actúen de maneras distintas. Los modelos construyen y condicionan identidades pero no pueden determinarlas en la praxis social. Este parámetro es otro de los que han ocultado históricamente la participación sociopolítica femenina.

El tercer argumento utilizado para negar la influencia de las mujeres ha sido su uso del hiyab. Esta prenda permitía avalar las propuestas anteriormente enunciadas de subordinación masculina, ausencia de voz, negación de sus poderes, etc, dado que posibilitaba hacer visible su negación o rechazo como sujeto social activo. Al tiempo, relacionaba a las mujeres con ciertas características que legitimarían esta invisibilidad y señalaría la coartada de su exclusión: sometimiento, silencio, diletancia, obediencia..., aspectos que casi venían a justificar las razones por las que era mejor que se obviase su posible participación sociopolítica: su dependencia. Ésta precisamente señalaría su incapacidad de tomar parte en las decisiones que afectasen al grupo ya que las mujeres no eran autónomas, independientes y objetivas como podían serlo los hombres.

\section{MUJERES VISIBLES: OTRAS MIRADAS SOBRE LA PARTICIPACIÓN POLITIICA DE LAS MUJERES}

La década de los años noventa representó un importante punto de inflexión respecto a los análisis que se estaban desarrollando en torno a la participación política de las mujeres en el mundo árabe. Las influencias fueron diversas pero sobre todo imperó el hecho de que los científicos sociales, desde sus respectivas disciplinas, revisasen la manera en que se venía analizando la influencia social de las mujeres. No debe olvidarse tampoco que estas nuevas perspectivas venían influidas por dos hechos diversos que impactaron de manera notable en la producción científica que se estaba desarrollando: el feminismo de los años setenta a partir del trabajo de Simone de Beauvoir y la insistente notoriedad de las mujeres en partidos políticos de distintos países, así como su asociacionismo y visibilidad pública en lugares como Egipto, Palestina o Argelia ${ }^{23}$.

22. Véase Bourdieu, Pierre: Esquisse d'une théorie de la pratique, Geneva, Librairie Droz, 1972.

23. Incluso en otros lugares del mundo musulmán como Irán o Turquía, lo que se observa en las obras de ADELKHAH, Fariba: La revolución bajo el velo. Mujer iraní y régimen islamista, Barcelona, Ediciones Bellaterra, 1996 (1991); MiR-HosseInI, Ziba: «Stretching the Limits: A Feminist Reading of the Sharia in Post- Khomeini Iran" en Mai Yamani, (ed.): Feminism and Islam: Legal and Literary Perspectives, New York, New York University Press, 1996, pp. 285-319; o también en el trabajo de NAJMABADI, Afsaneh: "Feminisms in an Islamic Republic: Years of Hardship, Years of Growth", en Y. Haddad y J. Esposito (eds.): Islam, Gender and Social Change, Oxford, Oxford University Press, 1998, pp. 5984; Sullivan, Zohreh T.: «EEludir a la feminista, desbancar lo moderno? Transformaciones en Irán durante el siglo XX» en L. Abu-Lughod (ed.): Feminismo y modernidad..., op. cit., pp. 315-354. 
No nos detendremos en ambos aspectos, sino en las implicaciones que tuvo el cambio de mirada por parte de los investigadores: es constatable que empiezan a emerger investigaciones que, en contra de la propuesta "oficial» anteriormente analizada, señalan los espacios de influencia que las mujeres han ostentado en sus países o la manera en que éstas han influido en la construcción de los estados independientes del siglo $\mathrm{XX}^{24}$. Es más, parte de estos estudios hacen hincapié en que esta visibilidad podría reconstruirse históricamente y sumergirse no sólo en la historia contemporánea, sino también en la moderna.

De hecho, algunos de los argumentos a los que se recurre para visibilizar a las mujeres a veces coinciden con algunos de los más utilizados para rechazar la participación social femenina: el hiyab. Para algunos investigadores éste no simboliza la pasividad femenina, al contrario, defienden que un sector del colectivo de mujeres en todos los países se ha reapropiado de él en el siglo XX para señalar precisamente cuál era su opción sociopolítica de futuro: la reproducción de la sociedad musulmana. Incluso, apuntan la estrecha vinculación entre algunos movimientos feministas e islamistas en la esfera política ${ }^{25}$. Este hecho desconcierta a algunas estructuras europeas dado que la democracia como sistema político parece que tiene dificultades en emerger bajo el paraguas de un islam que se designa de manera reiterada como patriarcal: ¿cómo es posible que haya mujeres que militen en política y porten el baluarte del islam? ¿hasta qué punto, una mujer que vista el hiyab es una mujer políticamente libre?

En cualquier caso, cabe apuntar que parte de estos estudios enuncian la necesidad de trabajar nuevamente el concepto "feminismo" dado que las propias sociedades árabes del mundo musulmán están señalando a través de su praxis cotidiana la existencia de discursos distintos y dispares respecto a cuál debe ser el lugar que las mujeres deben ocupar en la esfera social.

\section{CONCLUSIONES}

El texto que les presentamos ha pretendido hacer más próximo el debate existente sobre mujeres y política en el mundo árabe, resaltando la complejidad del tema. Uno de los objetivos, creemos cumplido, era el de mostrar que no se trata de un debate cerrado ya que puede y debe ser analizado desde diferentes prismas. De hecho, una de las dificultades de abordar esta temática manifiesta en el propio desarrollo de este artículo, ha sido que no sólo debíamos desentrañar la participación de las mujeres dentro de la esfera política, intentado aislarlas de los estereotipos que preconcebían su incapacidad de acción, sino que ni siquiera estaba consensuada la posibilidad de considerar estos regímenes polí-

24. BADRAn, Margot: "Competing Agenda: Feminist, Islam and the State in Ninetieth-Century Egypt», en Women, Islam \& State, London, Mcmillan, 1991, pp. 1-21; Aixela Cabré, Yolanda: Op. cit.; ABu-Lughod, Lila: «Zones of Theory...», Op. cit., y ABu-Lughod, Lila: «Introducción. Anhelos feministas...», op. cit.

25. Véase ABu-Lughod, Lila: «El matrimonio del feminismo y el islamismo en Egipto: el repudio selectivo como dinámica de la política cultural postcolonial» en L. Abu-Lughod (ed): Feminismo y modernidad..., op. cit., pp. 355-394. 
ticos como democráticos ni tampoco la existencia o no de una "sociedad civil» en su seno. A ello se ha sumado la habitual confusión entre «islam» y "árabe», y por supuesto, el que se plantease de manera constante una cierta homogeneidad de toda la región en términos, no ya políticos, sino religiosos.

Por todo ello, este artículo ha querido plantear la diversidad de opiniones existente entre los científicos sociales sobre «lo político» en el mundo árabe y sobre "la presencia o ausencia» del colectivo femenino en esta esfera. Como hemos observado, los trabajos reseñados en algunas ocasiones han abordado el tema comparándolo, consciente o inconscientemente, con las sociedades europeas, lo que ha confirmado algunas opiniones escépticas respecto a la inviabilidad del sistema democrático en el marco arabo-musulmán. No obstante, la revisión histórica de las sociedades arabo-musulmanas realizada por otros investigadores también citados nos ha permitido poner de manifiesto sus actuaciones y especificidades, abriendo nuevos interrogantes sobre el devenir de la democracia y de los feminismos en estos países.

En cualquier caso, deseamos terminar señalando una constatación fruto de este esfuerzo de síntesis: el debate apriorístico existente en el análisis de «lo político", por un lado, y de "las mujeres", por el otro, afecta directamente cuando se analizan ambos factores en común, en tanto que la consideración de la "política» en fricción con "lo democrático», con «lo religioso» y con "la sociedad civil», y de «las mujeres» con "lo patriarcal»y "lo religioso» genera toda una serie de distorsiones implícitas que van a dificultar que el investigador se aproxime a unas prácticas sociopolíticas «reales» que se alejen de prejuicios y estereotipos culturales, y también a su posibilidad de proponer nuevas perspectivas sobre el desarrollo político de los estados árabes y, cómo no, de la participación social de las mujeres que los habitan. 